\title{
The Use of Salivary Gland Scintigraphy for Diagnosis of Primary Sjögren Syndrome and Thyroid Disease in Patients with Dry Mouth
}

\author{
Keun Jeong Park, Bok Eum Kim, Jung Eun Lee, YounJung Park, \\ Jeong-Seung Kwon, Hyung-Joon Ahn, Jong-Hoon Choi
}

Department of Orofacial Pain and Oral Medicine, Dental Hospital of Yonsei University College of Dentistry, Seoul, Korea

Received November 28, 2019

Revised December 10, 2019

Accepted December 10, 2019

Correspondence to:

Jong-Hoon Choi

Department of Orofacial Pain and Oral

Medicine, Dental Hospital of Yonsei

University College of Dentistry, 50-1 Yonsei-

ro, Seodaemun-gu, Seoul 03722, Korea

Tel: +82-2-2228-3113

Fax: +82-2-393-5673

E-mail:JHCHOIJ@yuhs.ac

https://orcid.org/0000-0003-3211-3619
Sjögren syndrome (SS) is a chronic autoimmune disease characterized by dryness of the mouth and eyes due to lymphocytic infiltration of the exocrine glands. In American European Consensus Group (AECG) criteria, abnormal salivary gland scintigraphy (SGS) result is one of the objective signs of SS and it has been proposed as a valid and non-invasive alternative approach to functional evaluation of salivary gland, especially in the case when unstimulated whole salivary flow is more than $1.5 \mathrm{~mL}$ in 15 minutes or other AECG criteria is unmet. Patients with SS are more likely to have the thyroid disease (TD), but this association remains controversial. We present a case of the use of SGS for diagnosis of primary SS and TD in patients with dry mouth and burning sensation of tongue. Through this case, we suggest the usefulness of salivary scintigraphy for screening TD in addition to diagnosis of SS.

Key Words: Burning mouth syndrome; Radionuclide imaging; Sjogren's syndrome; Thyroid diseases; Xerostomia

\section{INTRODUCTION}

Sjögren syndrome (SS) is a chronic autoimmune disease characterized by dryness of the mouth and eyes due to lymphocytic infiltration of the exocrine glands [1]. It may occur alone as primary Sjögren syndrome (pSS) or be associated with other connective tissue diseases as secondary SS. Although the recent diagnostic criteria for SS are American College of Rheumatology/European League Against Rheumatism (ACR/EULAR, 2016) criteria American European Consensus Group (AECG, 2002) criteria are still used in National Health Insurance policy for incurabledisease. The AECG criteria are widely accepted which are based on a positive response to a short questionnaire of ocular and oral symptoms, ocular signs, histopathology, oral signs including at least one of these 3, unstimulated whole gland salivary flow $(\leq 0.1 \mathrm{~mL} / \mathrm{min})$, abnormal parotid sialography or abnormal salivary gland scintigraphy (SGS), and autoantibodies serum. For the diagnosis of pSS, any 4 of these 6 criteria can be used, provided that histopathology or autoantibodies are included or any 3 of the above criteria provided that histopathology, autoantibodies, unstimulated whole salivary flow, SGS or sialography are included $[2,3]$. The result of SGS is one of the objective signs of SS, providing a detailed functional evaluation of salivation. It measures the amount and speed of radioisotope uptake and excretion for all four major salivary glands separately [4]. The most common scintigraphic abnormality observed in SS, is the impairment of excretion, followed by a decrease in tracer accumulation, reflecting glandular parenchyma destruction [5]. So it is useful assessment method for patients who complain of dry mouth although their unstimulated salivary flow rate is more than $0.1 \mathrm{~mL} / \mathrm{min}$ or other AECG criteria is unmet.

Copyright (c) 2019 Korean Academy of Orofacial Pain and Oral Medicine. All rights reserved.

(c) This is an open-access article distributed under the terms of the Creative Commons Attribution Non-Commercial License (http://creativecommons.org/licenses/by-nc/4.0/), which permits unrestricted non-commercial use, distribution, and reproduction in any medium, provided the original work is properly cited. 
In this study, we present the case of pSS and thyroid disease (TD): 58-year-old woman with dry mouth and burning sensation of tongue who was diagnosed with pSS, hypothyroidism and goiter through SGS.

\section{CASE REPORT}

A 58-year-old woman patient had chief complaints of dry mouth, burning sensation of tongue, and recurrent oral ulcer. The onset of dry mouth and burning pain was about 2 months ago and oral ulcer was about 3-4 days ago. She had been complaining of spontaneous pain on tongue tip like burning sensation (VAS 2-3) and it was aggravated by fatigue.

In the first visit, the patient had ulcerative lesion on the right lower vestibule area but no oral lesion on the tongue. She also complained of dry mouth and dry eyes. On secretary function test (total saliva) salivation was decreased slightly as $0.167 \mathrm{~mL} / \mathrm{min}$. Laboratory tests including complete blood count, routine chemistry, serum iron, total iron binding capacity, ferritin, C-reactive protein, erythrocyte sedimentation rate (ESR), Vitamin B12, folic acid, ferritin, zinc, thyroid hormones $\mathrm{T} 3$ and $\mathrm{T} 4$, thyroid stimulating hormone (TSH), antinuclear antibody, anti-SS-A/Ro and anti-SS-B/La were carried out. It revealed that level of ESR and TSH was significantly high and free-T4 was significantly low. As anti-SS-A/Ro revealed positive indicating high possibility of pSS, SGS was done for national health insurance benefit diagnosed with pSS in the second visit (7 weeks after the first visit). The scintigraphic abnormality was observed as decrease in tracer accumulation on left submandibular gland and bilateral parotid glands. And significantly enlarged bilateral thyroid glands with increased radiotracer uptake was also observed (Fig. 1).

In the third visit, she was prescribed pilocarpine, and referred to endocrinology for thyroid evaluation. In the department of the endocrinology, sonography was done. And it revealed a low suspicious nodule on Left isthmus and
(A)

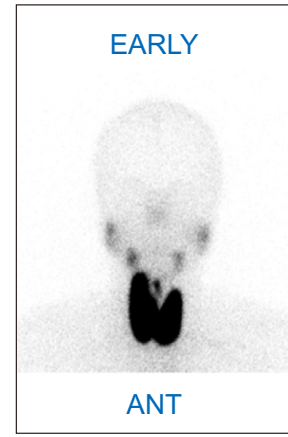

(B)

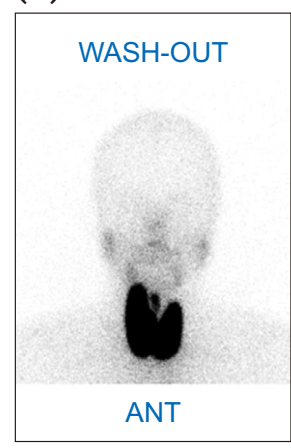

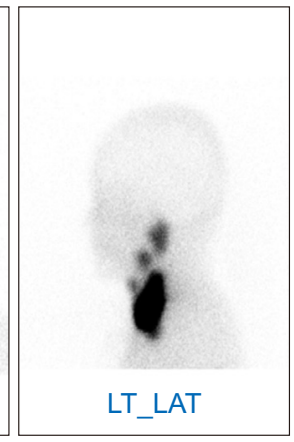

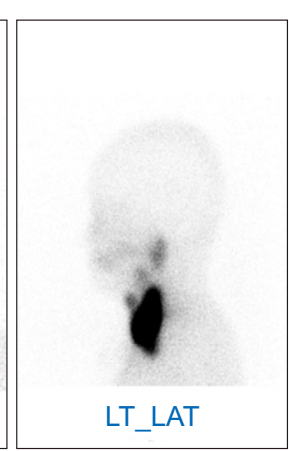

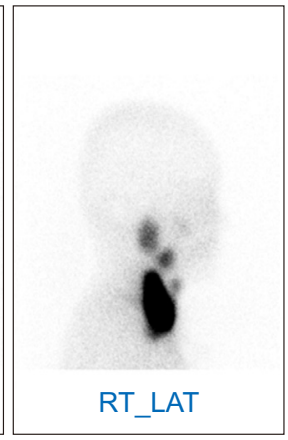

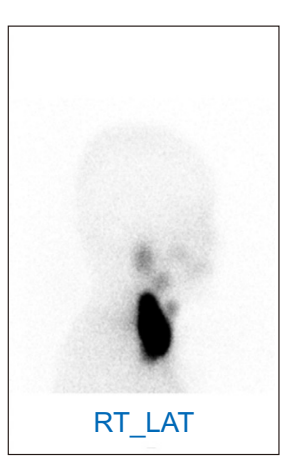

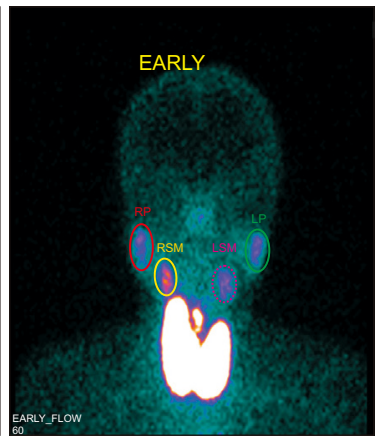
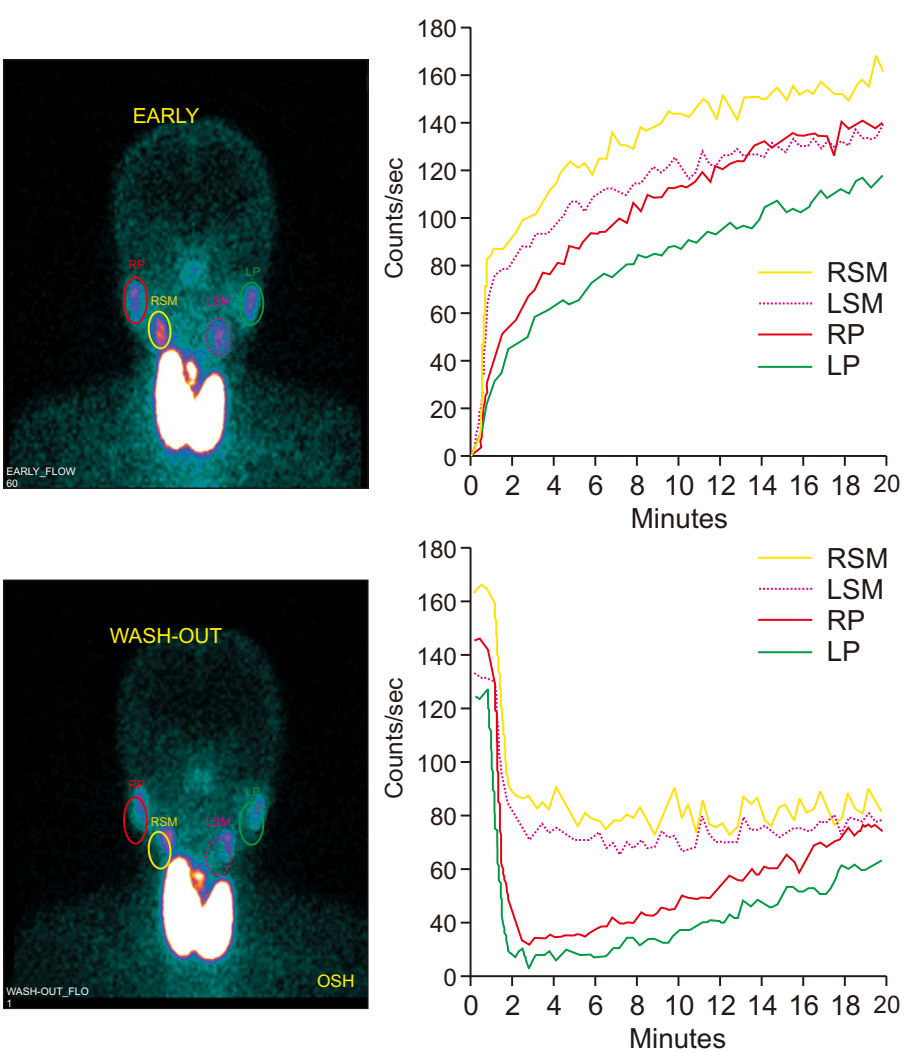

Fig. 1. Results of salivary gland scintigraphy. (A) A 5 minute after injection. (B) After stimulation. The images show the accumulation of saliva after Tc99m was injected, as well as release of saliva after stimulation. It shows that uptake to the left submandibular and bilateral parotid glands is decreased, and Enlarged bilateral thyroid glands with increased radiotracer uptake. ANT, anterior; LT, left; LAT, lateral; RT, right; RSM, right submandibular; LSM, left submandibular; RP, right parotid; LP, left parotid. 

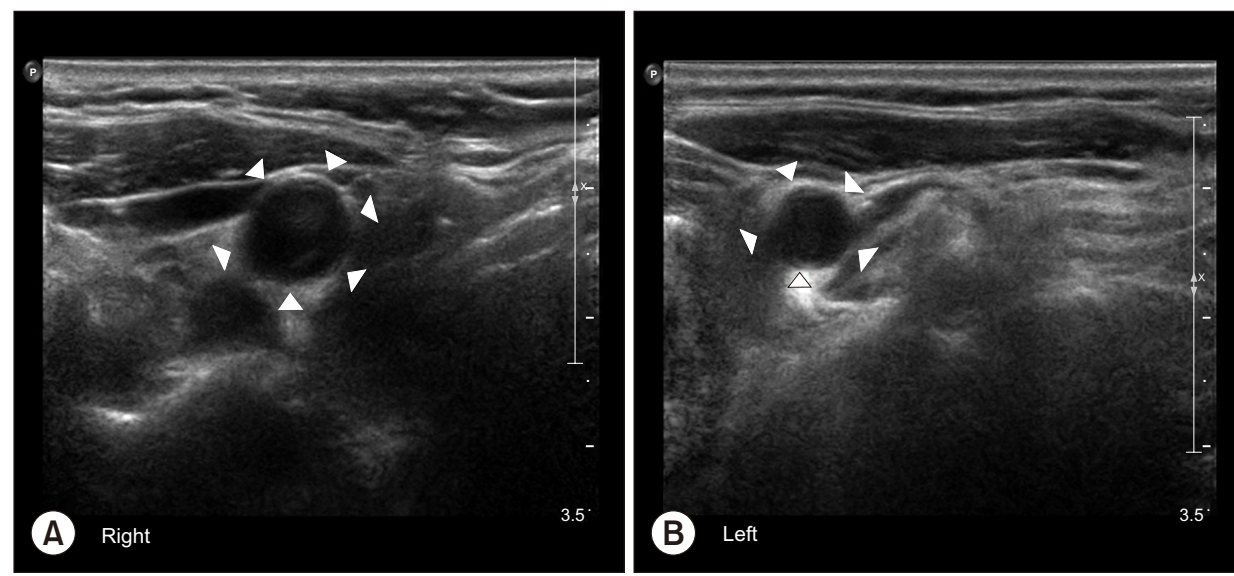

Fig. 2. Results of thyroid gland sonography. (A) Right thyroid gland, (B) left thyroid gland. Arrowheads indicate probably benign nodules on both thyroid gland, respectively. It shows enlarged bilateral thyroid glands with heterogeneous echogenicity.

several probably benign nodules on both (Fig. 2). She was prescribed synthyroxine diagnosed with hypothyroidism and goiter by endocrinologist.

In the fourth visit, she didn't complain of oral pain and dry mouth and the unstimulated salivary flow rate became normal. In the fifth visit, increased salivary flow rate was maintained after stop taking the pilocarpine.

\section{DISCUSSION}

SS is an autoimmune disease that mainly affects the exocrine glands and induces functional impairment of salivary gland and lacrimal gland presenting as dry mouth and dry eyes. It may occur alone as pSS or be associated with other connective tissue diseases as secondary SS [6].

Thyroid, salivary, and lacrimal glands are similar from a histological and functional point of view. This suggests that autoimmune TD and SS can be related immunologically each other. Although association of TD with SS remains controversial, Hashimoto's thyroiditis with SS was recently confirmed [6]. The prevalence of Hashimoto's thyroiditis in SS was higher than in the general population [7]. Patients with pSS also have an increased tendency to develop other autoimmune disease like autoimmune TD, and the most frequent hormonal profile is subclinical hypothyroidism [8]. In regard to the correlation of TD with SS, one-third of patients with TD may have symptom of xerostomia and approximately 10\% of patients with anti-nuclear antibody positive autoimmune thyroiditis fulfill the criteria for the diagnosis of SS $[9,10]$. This suggests that the symptom of dry mouth should be considered as possible sign of TD [11].
In addition, the level of anti-TP0, anti-TG, free T3, free T4 and TSH indices is associated with burning mouth syndrome in patients with Hashimoto's thyroiditis. Especially hypothyroidism is one of the factors affecting burning sensation [12]. Therefore, if patients with burning mouth syndrome visit the clinic, clinicians should consider the possibility of TD and check symptoms including dry mouth.

SGS, as a diagnostic tool in patients with SS, may be helpful for screening TD. This mothed is accepted by AECG criteria for SS as objective quantitative evaluation of salivary gland function. In time-activity curve from scintigraphy results, excretion fraction (EF\%) calculated as [maximum activity - minimum activity / maximum activity] for each major salivary gland is important value of function assessment. EF\% is significantly reduced in patients with dry mouth, especially more than moderate lymphocyte infiltration of salivary gland tissue in result of minor salivary gland biopsy, compared with less than mild lymphocyte infiltration [2].

The advantages of scintigraphy compared with other methods, such as computed tomography, magnetic resonance imaging, sialography and sialometry, are non-invasive, reproducible and easy to perform. Most of all, quantitative assessment of SGS is sensitive enough to detect excretion abnormalities not only major salivary glands but also thyroid glands [5,13]. In the way that AECG criteria are still used in National Health Insurance policy for SS, SGS is useful for diagnosis.

In conclusion, SGS is worthy to be considered for diagnosis of SS when patients complain of dry mouth. The risk of TD in patients with pSS is significantly increased. Therefore, 
SGS can be used for early detection of TD especially to women with pSS.

\section{CONFLICT OF INTEREST}

No potential conflict of interest relevant to this article was reported.

\section{ORCID}

Keun Jeong Park

https://orcid.org/0000-0003-4559-9398

Bok Eum Kim

https://orcid.org/0000-0001-7396-2479

Jung Eun Lee

https://orcid.org/0000-0001-6439-8087

YounJung Park

https://orcid.org/0000-0002-9152-7849

Jeong-Seung Kwon

https://orcid.org/0000-0003-4584-7355

Hyung-Joon Ahn

https://orcid.org/0000-0001-9669-9781

Jong-Hoon Choi

https://orcid.org/0000-0003-3211-3619

\section{REFERENCES}

1. Dugonjić S, Stefanović D, Ethurović B, Spasić-Jokić V, Ajdinović B. Evaluation of diagnostic parameters from parotid and submandibular dynamic salivary glands scintigraphy and unstimulated sialometry in Sjögren's syndrome. Hell J Nucl Med 2014;17:116-
122.

2. Aksoy T, Kiratli PO, Erbas B. Correlations between histopathologic and scintigraphic parameters of salivary glands in patients with Sjögren's syndrome. Clin Rheumatol 2012;31:1365-1370.

3. Vitali C, Bombardieri S, Jonsson R, et al. Classification criteria for Sjögren's syndrome: a revised version of the European criteria proposed by the American-European Consensus Group. Ann Rheum Dis 2002;61:554-558.

4. Umehara I, Yamada I, Murata Y, Takahashi Y, Okada N, Shibuya H. Quantitative evaluation of salivary gland scintigraphy in Sjörgen's syndrome. J Nucl Med 1999;40:64-69.

5. Vinagre F, Santos MJ, Prata A, da Silva JC, Santos AI. Assessment of salivary gland function in Sjögren's syndrome: the role of salivary gland scintigraphy. Autoimmun Rev 2009;8:672-676.

6. Jara LJ, Navarro C, Brito-Zerón Mdel P, García-Carrasco M, Escárcega R0, Ramos-Casals M. Thyroid disease in Sjögren's syndrome. Clin Rheumatol 2007;26:1601-1606.

7. Biró E, Szekanecz Z, Czirják L, et al. Association of systemic and thyroid autoimmune diseases. Clin Rheumatol 2006;25:240-245.

8. Sun X, Lu L, Li Y, Yang R, Shan L, Wang Y. Increased risk of thyroid disease in patients with Sjogren's syndrome: a systematic review and meta-analysis. PeerJ 2019;7:e6737.

9. Mavragani CP, Fragoulis GE, Moutsopoulos HM. Endocrine alterations in primary Sjogren's syndrome: an overview. J Autoimmun 2012;39:354-358.

10. Tektonidou MG, Anapliotou M, Vlachoyiannopoulos P, Moutsopoulos HM. Presence of systemic autoimmune disorders in patients with autoimmune thyroid diseases. Ann Rheum Dis 2004;63:1159-1161.

11. Jung JH, Lee CH, Son SH, et al. High prevalence of thyroid disease and role of salivary gland scintigraphy in patients with xerostomia. Nucl Med Mol Imaging 2017;51:169-177.

12. Talattof Z, Dabbaghmanesh MH, Parvizi Y, Esnaashari N, Azad A. The association between burning mouth syndrome and level of thyroid hormones in Hashimotos thyroiditis in public hospitals in Shiraz, 2016. J Dent (Shiraz) 2019;20:42-47.

13. Afzelius P, Nielsen MY, Ewertsen C, Bloch KP. Imaging of the major salivary glands. Clin Physiol Funct Imaging 2016;36:1-10. 